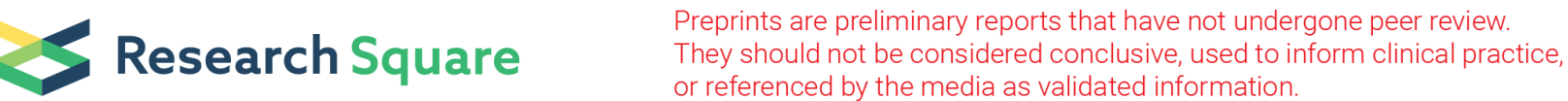

\section{ACDF Using a Zero Profile Cage Plate for The Management Of Degenerative Cervical Disc Disease, Early Experience From A Middle Eastern Tertiary Hospital.}

\section{Tamer Ramadan}

Alrazi Hospital

Ahmed A. Khalifa ( $\square$ ahmed_adel0391@med.svu.edu.eg )

South Valley University Faculty of Medicine https://orcid.org/0000-0002-0710-6487

Morsy Basiony

Aswan University

Mahmoud Abdelkarim

Cairo University

Ahmed Abdellatif

Aswan University

\section{Research Article}

Keywords: Anterior cervical discectomy and fusion, Intervertebral device, Zero profile plate, cervical disc disease, Cages

Posted Date: August 17th, 2021

DOl: https://doi.org/10.21203/rs.3.rs-809319/v1

License: (c) (i) This work is licensed under a Creative Commons Attribution 4.0 International License.

Read Full License 


\section{Abstract}

Background: Management of cervical disc degenerative diseases through anterior cervical decompression and fusion (ACDF) using various implants had been reported, a Zero-p device used as a stand-alone cage plate in ACDF was introduced to offer higher safety and efficacy. In the current prospective cohort study, we aimed at reporting our early experience from a Middle Eastern tertiary hospital after using the Zero profile cage plate in the management of degenerative cervical disc diseases through ACDF by reporting the clinical, radiological outcomes as well as the incidence of complications.

Methods: Thirty patients ( 16 male and 14 female) with a mean age of $47.9 \pm 10.9$ underwent ACDF with Zero-p implant, a total of 43 operated levels (one level in 20 patients, double levels in 7, and multi-levels in 3). Clinical outcomes were evaluated according to VAS to measure the neck and radicular arm pain and neck disability index (NDI). Radiographic bony fusion was assessed according to Pitzen criteria, any perioperative complications were reported, and the presence of dysphagia was assessed according to the Bazaz-Yoo dysphagia index.

Results: At a mean follow up of $14.4 \pm 2.3$ months, all patients obtained radiographic bony fusion, all patients had a significant reduction of neck and radicular arm pain with a mean VAS score of 7.33 and 8.70 preoperatively to 1.73 and 0.27 at 12 months follow-up respectively $(p<0.0001)$, and reduction of NDI from $68.87 \%$ preoperatively to $8.60 \%$ at 12 months follow-up ( $p<0.0001$ ). None had dysphagia after six months postoperatively; one patient developed back-out of one of the implant screws that was extracted, and otherwise, no other implant-related complications.

Conclusion: the Zero-p implant is a valid alternative to anterior cervical plating after ACDF with a very low incidence of chronic dysphagia and implant-related complications.

\section{Level of Evidence $\rrbracket$}

\section{Introduction:}

Neck pain resulting from the cervical spine's degenerative conditions, such as degenerative disc diseases or cervical spondylotic myelopathy accompanied by radicular pain and neurological deficits, could be resistant to conservative management lines and became major indications for anterior cervical discectomy and fusion (ACDF) (1).

Although ACDF proved to provide excellent long term results, however (2), there is still a debate regarding the optimum construct to be used to obtain fusion and the best functional and radiological results (1), as ACDF could be performed through different techniques with the help of various implants, as anterior interbody fusion using a plate for fixation and a supplemental iliac bone autograft, or the use of cages either as a stand-alone or augmented by anterior plate fixation (3-5). 
In order to obtain fusion, it is generally agreed that intervertebral motion should be minimized so bone growth can occur. Furthermore, an interbody graft or spacer position should be maintained to prevent its extrusion, irritation of surrounding tissues, and allow union with the adjacent vertebrae $(6,7)$.

Many surgeons prefer to add plate infusion procedures for enhancing stabilizing properties, as several studies suggested that this led to increased fusion rates with failure reduction (particularly in multilevel procedures) and less incidence of cervical kyphosis (8). However, adding a plate to the construct, even with low-profile implants, had been reported to cause some complications such as chronic dysphagia, which was reported to occur in an incidence of $3 \%$ to $21 \%(9,10)$. Cases of migrating screws and subsequent soft tissue damage were reported as well $(11,12)$.

To avoid the complications mentioned earlier of the plates, Zero-profile implants to be used as a standalone construct in ACDF were introduced, which proved efficacy and safety (13-16). The current study aimed to report our early experience from a Middle Eastern tertiary hospital after using a Zero-profile cage plate to manage degenerative cervical disc diseases through ACDF. Clinical and radiological outcomes, as well as the incidence of complications, were reported.

\section{Methods:}

This is a single-center prospective cohort study carried in the period between February 2017 to February 2019 after gaining approval from the institutional ethical committee and written informed consent from patients approving the participation in the study, a consecutive series of thirty patients underwent ACDF using the Zero-profile cage plate, patients aged between 18 to 65 years diagnosed with cervical disc disease at levels from $\mathrm{C} 3$ to $\mathrm{C} 7$ (diagnosis confirmed by the magnetic resonance imaging (MRI) study) with a cervical radicular syndrome (neck or arm radicular pain) and/or myelopathy failing conservative treatment with at least 12 months follow up were included. Patient having a systemic or local infection, active rheumatoid arthritis, or any other medical conditions that interfere with normal healing or increase surgical risk, previously known allergy to the materials contained in the device, such as polyether ketone or titanium alloy, and the traumatic lesion was excluded from having this surgery as well as being included in the study.

The Preoperative assessment of the patients consisted of clinical evaluation, which included a complete neurological examination (motor, sensory, and reflexes) and painful symptoms quantification using the visual analog scale (VAS) for neck and radicular pain (scale of 0 to 10 , where 0 means no pain and 10 means severe pain), and neck pain disability scale (NDI) ranging from $0-100 \%$ (17). While imaging studies were performed in the form of plain radiological studies (x-ray) of the cervical spine (Anteroposterior and lateral views) and MRI (T1 and T2-weighted images in sagittal and axial planes).

\section{Description of the implant used (Fig. 1):}

In this series, we used a Zero-profile cage plate (zero-p; Synthes $\mathrm{GmbH}$, Oberdorf, Switzerland) which acts as a stand-alone implant for cervical interbody fusion. Its design combines the functionality of a cervical 
interbody spacer and the benefits of an anterior cervical plate. The Zero-profile implant will be contained within the excised disc space and will not protrude past the vertebral body anterior wall as do anterior cervical plates and avoid these complications. The Zero-profile cage plate consists of a spacer component made of PEEK optima (polyetheretherketone), which contains carbon fibers reducing the risk of systemic uptake and local connective tissue formation, and teeth on the implant surface provide initial stability. Titanium alloy plate provides a secure, rigid screw locking interface, locking head screws with a $40 \pm 5^{\circ} \mathrm{cranial} / \mathrm{caudal}$ angle and $2.5^{\circ} \mathrm{medial} /$ lateral angle, self-tapping screws improve thread purchase. In February 2008, The US Food and Drug Administration (FDA) approved the clinical use of Zero Profile Cage plate (Zero-P) in skeletally mature patients for degenerative cervical spine conditions (18).

\section{Surgical technique:}

under general anesthesia, patients were placed supine on an operative table with a head extension in supine. After draping in the usual manner, the target disc space was approached through a standard anterior approach to the cervical spine after locating the disc space using an image intensifier (19).

Anterior decompression was performed first; then trial spacers were used to determine the proper implant size that would be used. After the trial spacer was correctly fitted into the disc space, a corresponding zero-p implant filled with a synthetic bone graft (demineralized bone matrix (DBM)) was inserted with an implant aiming device.

They integrated a small plate containing four holed with internal screw threads. After trilling the pilot hole through the aiming device, the first locking screw was inserted. The implant system contains screws of $12 \mathrm{~mm}, 14 \mathrm{~mm}$, and $16 \mathrm{~mm}$ lengths that the screw length was according to measurement, and in most of our cases, the $16 \mathrm{~mm}$ screw length was used. Subsequently, the other three holes were drilled using the guidance of the aiming device. The aiming device was then removed, and the remaining screws were inserted using torque limitation (1.2 Nm). Angled instruments for drilling and inserting screws in the upper and lower spine were used.

\section{Postoperative and follow up protocol:}

Immediately postoperatively, clinical (neurological) and radiological assessment was performed while the patient is in the recovery room. First-generation cephalosporin (cefazoline) antibiotic was prescribed for three days, and all patients were mobilized from the first day postoperatively. Miami collar was recommended postoperatively for 12 weeks, and x-ray A-P and lateral was repeated for all patients before discharge from the hospital.

Follow-up visits were scheduled at two weeks for a wound check and stitches removal, then at six weeks, three, six, nine, 12 months, and then annually. At each visit, clinical (full neurological assessment, VAS, and NDI) and radiological (plain radiographs AP and lateral) evaluation for assessment of implant position and bony fusion according to the criteria defined by Pitzen et al. (20) (the absence of a radiolucent gap between the graft and the endplates, the presence of continuous bridging trabeculae between the endplates and the absence of motion between the spinous processes on flexion/extension 
radiographs) were repeated, presence of dysphagia at any visit was assessed using and consisted of the Bazaz-Yoo dysphagia index (9). The outcomes at 12 months follow-up were reported, and any incident of complications (perioperative or during the follow-up visits) was reported as well.

\section{Statistical analysis}

Differences between preoperative and postoperative VAS, NDI, and Bazaz-Yoo scores were calculated using Freidman's test with posthic multiple 2-group comparisons. A P-value less than 0.05 was considered statistically significant. All statistical calculations were done using computer program SPSS (statistical package for the social science; SPSS Inc., Chicago, IL, USA) software version 15.0.1.

\section{Results:}

All patients were available for follow-up with a mean of $14.4 \pm 2.3$ months (range 12 to 18 ); the study cohort's basic demographic and surgical data are presented in (Table 1). Postoperative VAS was assessed for all patients for neck and radicular arm pain at each follow-up visit till 12 months follow up visit, all had a statistically significant reduction in VAS radicular pain $(p<0.0001)$ and neck pain $(p<$ 0.0001 ) within the first three months (Fig. 2A) and such improving trend was maintained at the following follow-up visits with reduction of the mean VAS score of the neck and radicular arm pain from 7.33 and 8.70 preoperatively to 1.73 and 0.27 at 12 months follow-up respectively. All patients had a statistically significant reduction in NDI in the first three months $(p<0.0001)$, and such a positive trend was maintained at the following follow-ups with a reduction of the NDI from $68.9 \%$ preoperatively to $8.6 \%$ at 12 months (Fig. 2B). All patients had radiological signs of fusion according to criteria reported by Pitzen et al. (20) (Fig. 3) by three months except one (3.3\%) patient who was followed monthly till fusion was obtained by the six months follow-up. No adjacent segment disease was noted radiographically till the last follow-up. Among the study population, 11 (36.7 \%) patients complained of postoperative dysphagia ranged from mild dysphagia (score 1) to moderate dysphagia (score 2), this number was reduced to 4 (13.3\%) patients at 1-month follow-up and to only one (3.3\%) patient at three months follow-up, no patient complained of dysphagia at six months follow up. One (3.3\%) patients developed an insidious onset of moderate dysphagia at three months follow up after being free from any symptoms; further assessment of this patient revealed screw back-out (the operated level was fused) due to failure of the locking mechanism (Fig. 4), symptoms of dysphagia disappeared after revision surgery to extract the screw. No other postoperative complications were recorded as wound infection, hoarseness of voice, neurological or vascular injures, or further implant-related complications such as subsidence, screw cutthrough, or cage dislodgment. 
Table 1

Demographic and surgical data of the study cohort.

\begin{tabular}{|c|c|c|}
\hline \multicolumn{3}{|c|}{ Demographic Variables of Total $30(100 \%)$ patients } \\
\hline \multicolumn{2}{|l|}{ Age* $^{*}$} & $47.9 \pm 10.9$ years ( 31 to 65 ) \\
\hline \multirow{2}{*}{\multicolumn{2}{|c|}{ Gender** }} & $16(53.3 \%)$ males \\
\hline & & $14(46.7 \%)$ females \\
\hline \multicolumn{2}{|l|}{ Hospital stay* } & $3.8 \pm 1.2$ days ( 3 to 7 ) \\
\hline \multicolumn{2}{|l|}{ Follow-up* } & $14.4 \pm 2.3$ months ( 12 to 18 ) \\
\hline \multicolumn{3}{|l|}{ Surgical data } \\
\hline \multicolumn{2}{|l|}{ Blood loss* } & $206.7 \pm 85 c c$ \\
\hline \multicolumn{2}{|l|}{ Operative time* } & $131.8 \pm 35$ minutes ( 90 to 220 ) \\
\hline \multicolumn{3}{|l|}{ Operated levels** } \\
\hline \multirow{3}{*}{$\begin{array}{l}\text { Total } \\
30(100 \%) \text { patients }\end{array}$} & Single level & $20(66.7 \%)$ patients \\
\hline & Double levels & seven $(23.3 \%)$ patients \\
\hline & Multiple levels & Three (10\%) patients \\
\hline \multirow[t]{4}{*}{ Total $43(100 \%)$ levels } & C3-C4 & $3(7 \%)$ \\
\hline & C4-C5 & $11(25 \%)$ \\
\hline & $\mathrm{C} 5-\mathrm{C} 6$ & $21(49 \%)$ \\
\hline & $\mathrm{C6}-\mathrm{C} 7$ & $8(19 \%)$ \\
\hline \multicolumn{3}{|l|}{ Implant sizes** } \\
\hline \multirow[t]{4}{*}{ Total 43 (100\%) levels } & $5 \mathrm{~mm}$ & $1(2 \%)$ \\
\hline & $6 \mathrm{~mm}$ & $4(10 \%)$ \\
\hline & $7 \mathrm{~mm}$ & $21(49 \%)$ \\
\hline & $8 \mathrm{~mm}$ & $17(40 \%)$ \\
\hline
\end{tabular}

\section{Discussion:}

In the current limited series, after using the Zero-p device for ACDF as an option for management of cervical disc degenerative diseases, we could obtain acceptable functional outcomes, and radiographic bony fusion rate in the operated levels by the last follow up as well as a high margin of safety regarding the incidence of dysphagia and absence of series implant-related complications. 
After failed conservative lines, operative management of degenerative cervical disc disease consists mainly of either disc replacement surgery or ACDF, which could be performed through different techniques commonly using a plate and a cage or graft (21).

An ideal ACDF technique should achieve success in terms of fusion rate, neurological recovery, and fewer complication rates, aiming to maintain cervical alignment, eliminate potential instability, preserve disc height, and reduce nerve roots' compression (13).

Preservation of the disc space height could be achieved either by using an autogenous iliac bone graft with its known donor site comorbidity or using cervical cages to avoid the bone graft drawbacks. Furthermore, cage construct was found to achieve internal fixation while permitting bone formation in and around the device $(22,23)$.

The addition of plate reduces the incidence of graft and cage complications, acting as a buttress prevents graft extrusion preventing graft collapse and subsidence, and maintaining cervical lordosis, results in a higher incidence of fusion, less incidence of pseudoarthrosis, especially in multilevel ACDF $(14,24)$.

However, complications reported concerning the use of plate such as chronic dysphagia with a frequency of 2 to $67 \%$, implant-related complications, and neurovascular damage $(9,25)$, led to the development of a new low profile and stand-alone devices like the Zero-p devices, which avoid the contact with the anterior soft tissue structures leading to less irritation and adhesions, it also contained within the disc space, so it avoids mechanical irritation to the esophagus leading to less dysphagia incidence (26-28).

The functional improvement after using the Zero-profile device was nearly the same as obtained after ACDF using a plate and cage, as proved in a retrospective case-control study by Yang et al. with a mean follow up about 30 months comparing the Zero profile device (30 patients) to the anterior cervical plate and cage (32 patients), they found no significant statistical difference in the clinical outcomes according to Japanese Orthopaedic Association (JOA) scores between both techniques, there were significantly better postoperatively compared to preoperatively in both groups $(p<0.05)(16)$.

In the current series where we operated on $20(66.7 \%)$ one level, double levels in seven patients, and multilevels in three, all patients obtained a significant reduction in neck and radicular arm pain by a mean decrease of three and five points as measured by VAS for both components of pain respectively; this improvement was comparable to the results obtained from a prospective study by Scholz et al. including 34 patients diagnosed with degenerative cervical disc diseases accompanied with radiculopathy or myelopathy who underwent ACDF using a Zero-profile Implant ( 15 one level, 20 double levels, and three multi-levels), all patients had a reduction in the VAS radicular arm and neck pain compared to the preoperative values $(p<0.001)$ during the first three months ${ }^{(15)}$.

There was a marked improvement in functional outcome assessment using NDI score in our patients, from a mean of $69 \%$ preoperatively to $31 \%$ postoperatively; this improvement continued till 12 months 
follow-up where it reached $8 \%$; this improvement was comparable to the results from a prospective study by Barbagallo et al. evaluating 32 patients with a mean follow-up of 27.3 months who had ACDF using a zero-profile cage-plate device for managing cervical disc degenerative disease (One level in 7 patients, two in 12, and multi-levels in 13), where they reported a statistically significant improvement in NDI postoperatively $(p<0.01)$ compared to preoperative values $(14)$.

In this study, all patients had radiological signs of fusion by three months (96.6\%) except for one patient who had a fusion at six months follow up; this was comparable to other studies where bony fusion was obtained in all patients as in the studies by Scholz et al. (15), and Azab W et al. (13), while Barbagallo et al. obtained fusion in $94.5 \%$ of their patients at three months follow-up (14).

In the study by Yang et al., the incidence of dysphagia measured according to the Bazaz-Yoo dysphagia index was lower with fewer symptoms and shorter duration in the Zero-p group compared to the plate and cage group ( $p<0.01$ ) (16). Postoperative dysphagia occurred at an incidence of $36.6 \%$ (11 patients) in the current study, mild in four (13.4\%), and moderate in 7 (23.2\%) patients; however, all patients had not complained of dysphagia by the six months follow up, the incidence reported in our study was less than previous studies where an incidence of $62 \%$ and $76 \%$ were reported in the studies by Scholz et al.(15) and Azab w et al.(13) respectively. Barbagallo et al. suggested that dysphagia was related to operating on multi-levels and occurred more with cranial levels (i.e., C3-C4), as eight patients out of $11(72.7 \%)$ who suffered from postoperative dysphagia in their series had multilevel ACDF (14).

In this study, there were no implant-related complications except one (3.3\%) screw back-out in a healthy young patient, which was reported at three months follow-up and was attributed to the failure of the locking mechanism of one of the implant's screws; this incident was similar to a reported case in the study by Barbagallo et al., where screw back-out occurred at one-month follow-up in a patient who had bad bone quality (14). However, in ACDF with anterior plating, an incidence of screw and plate loosening between $0-15.4 \%$, screw breakage between $0 \%$ and $13.3 \%$, plate breakage between $0 \%$ and $6.7 \%$, plate and graft displacement between $0 \%$ and $21.4 \%$, and implant malposition $0 \%$ and $12.5 \%$ as was reported by Vaccaro et al. (29). In the studies by Scholz et al. (15) and Yang et al. (16), there were no Zero-p implant-related complications; they referred the lack of implant migration or screw loosening in their studies to the design of locking plate-screw interface of the zero-p implant; the plate with an internal screw thread engages with the outer screw thread located in the head of the screw providing a safe, constrained, and angle-stable screw fixation.

We did not report surgery-related complications as postoperative hematoma, hoarseness of voice, and superficial infection. Postoperative hematoma that needs surgical evacuation was recorded in one patient in the study by Barbagallo et al. (14) and two patients in the study by Scholz et al. (15).

Reduced risk of inducing adjacent level degeneration and spondylotic changes was an advantage suggested with the use of zero-p devices; as it has been shown that cervical plates reaching the adjacent disc levels can induce and accelerate disc degeneration and osteophyte formation while the zero-p minimizes such risk as it remains within the disc space, far from adjacent-level disc spaces (14), in our 
study no occurrence of adjacent level segments detected till the last follow up, the same observation was reported in the study by Yang et al. where adjacent-level disease progression was detected in $12(18.8 \%)$ patients in the plate with cage group, and in only 1 (1.6\%) in Zero-p group, the difference was statistically significant $(p<0.01)(16)$. Another advantage of using the zero-p which is saving the in-between nondiseased levels during surgery, i.e., for example, a diseased C3-C4 segment and a diseased C5-C6 segment, and not to include the in-between C4-C5 non-diseased level with the less operative time needed than conventional plate and cage, in addition, revision, if needed, can be done for each level separately in multilevel fusion without attacking the other operated levels $(16,30)$. A shorter operative time in comparison to the traditional cervical cage and plate ACDF, with less time exposure for soft tissue retraction during the surgery, was suggested as another advantage by some authors $(13,14,24)$.

Our study had some limitations; first, it was performed as an observational study without including another control group (graft/cage and plate) for comparison. Second, the relatively small sample size in comparison to other studies. Third, a longer follow is needed to confirm the results obtained and evaluate adjacent level degeneration incidence.

In conclusion, a Zero-profile cage plate is a valid alternative to anterior cervical plating in patients undergoing ACDF and characterized by the low incidence of chronic dysphagia owing to the 'zero' implant profile and low incidence of implant-related complications.

\section{Declarations}

Ethical approval and Consent to participate: This article does not contain any experimental studies with human participants or animals performed by any of the authors, and the ethical committee of our institution approved it. Informed consent: obtained from all patients to use their radiographs for this research.

\section{Consent for publication: N/A}

Availability of data and material: All the data related to the study are mentioned within the manuscript, however, the raw data are available with the corresponding author and will be provided up on a written request.

Competing Interest: The authors declare that they have no conflict of interest.

Funding: This research did not receive any specific grant from funding agencies in the public, commercial, or not-for-profit sectors.

\section{Authors contribution:}

A.A. and T.R. carried out the Study conception and design and performed the surgeries, M.B. and M.A. carried out data acquisition, assessment, and measurements. A.A.K., and T.R. carried out analysis and interpretation of data, drafted the manuscript, and designed the figures and tables, A.A., M.B. and M.A. did 
the critical revision. All authors discussed the results and commented on the manuscript. All authors read and approved the final manuscript.

\section{No Acknowledgements}

\section{Abbreviations}

anterior cervical discectomy and fusion (ACDF)

magnetic resonance imaging (MRI)

visual analog scale (VAS)

neck pain disability scale (NDI)

polyetheretherketone (PEEK)

Food and Drug Administration (FDA)

demineralized bone matrix (DBM)

statistical package for the social science (SPSS)

\section{References}

1. Ahmed AF, Al Dosari MAA, Al Kuwari A, Khan NM. The outcomes of stand alone polyetheretherketone cages in anterior cervical discectomy and fusion. International orthopaedics. 2021;45(1):173-80.

2. Palma L, Mariottini A, Carangelo B, Muzii VF, Zalaffi A. Favourable long-term clinical outcome after anterior cervical discectomy. A study on a series of 125 patients undergoing surgery a mean of 11 years earlier. Acta Neurochir. 2010;152(7):1145-52.

3. Lord EL, Cohen JR, Buser Z, Meisel HJ, Brodke DS, Yoon ST, et al. Trends, Costs, and Complications of Anterior Cervical Discectomy and Fusion With and Without Bone Morphogenetic Protein in the United States Medicare Population. Global spine journal. 2017;7(7):603-8.

4. Bakhsheshian J, Mehta VA, Liu JC. Current Diagnosis and Management of Cervical Spondylotic Myelopathy. Global spine journal. 2017;7(6):572-86.

5. Shao MH, Zhang F, Yin J, Xu HC, Lyu FZ. Titanium cages versus autogenous iliac crest bone grafts in anterior cervical discectomy and fusion treatment of patients with cervical degenerative diseases: a systematic review and meta-analysis. Curr Med Res Opin. 2017;33(5):803-11.

6. Wen Z, Lu T, Wang Y, Liang H, Gao Z, He X. Anterior Cervical Corpectomy and Fusion and Anterior Cervical Discectomy and Fusion Using Titanium Mesh Cages for Treatment of Degenerative Cervical Pathologies: A Literature Review. Medical science monitor: international medical journal of experimental clinical research. 2018;24:6398-404. 
7. Zhang D, Liu B, Zhu J, Li C, Wei F, Yuan Y, et al. Comparison of Clinical and Radiologic Outcomes Between Self-Locking Stand-Alone Cage and Cage with Anterior Plate for Multilevel Anterior Cervical Discectomy and Fusion: A Meta-Analysis. World neurosurgery. 2019;125:e117-e31.

8. Duan Y, Yang Y, Wang Y, Liu H, Hong Y, Gong Q, et al. Comparison of anterior cervical discectomy and fusion with the zero-profile device versus plate and cage in treating cervical degenerative disc disease: A meta-analysis. Journal of clinical neuroscience: official journal of the Neurosurgical Society of Australasia. 2016;33:11-8.

9. Bazaz R, Lee MJ, Yoo JU. Incidence of dysphagia after anterior cervical spine surgery: a prospective study. Spine. 2002;27(22):2453-8.

10. Rosenthal BD, Nair R, Hsu WK, Patel AA, Savage JW. Dysphagia and Dysphonia Assessment Tools After Anterior Cervical Spine Surgery. Clinical spine surgery. 2016;29(9):363-7.

11. Yee TJ, Swong K, Park P. Complications of anterior cervical spine surgery: a systematic review of the literature. Journal of spine surgery (Hong Kong). 2020;6(1):302-22.

12. Tasiou A, Giannis T, Brotis AG, Siasios I, Georgiadis I, Gatos H, et al. Anterior cervical spine surgeryassociated complications in a retrospective case-control study. Journal of spine surgery (Hong Kong). 2017;3(3):444-59.

13. Waleed A, ABDEL-RAZEK M, Abeer A, ABDELRAHMAN A, SALAHELDIN W, NASIM K, et al. Outcome Evaluation of a Zero-Profile Implant for Anterior Cervical Diskectomy with Fusion. Turkish neurosurgery.22(5).

14. Barbagallo GM, Romano D, Certo F, Milone P, Albanese V. Zero-P: a new zero-profile cage-plate device for single and multilevel ACDF. A single institution series with four years maximum follow-up and review of the literature on zero-profile devices. European spine journal: official publication of the European Spine Society, the European Spinal Deformity Society, and the European Section of the Cervical Spine Research Society. 2013;22 Suppl 6(Suppl 6):S868-78.

15. Scholz M, Schnake KJ, Pingel A, Hoffmann R, Kandziora F. A new zero-profile implant for stand-alone anterior cervical interbody fusion. Clin Orthop Relat Res. 2011;469(3):666-73.

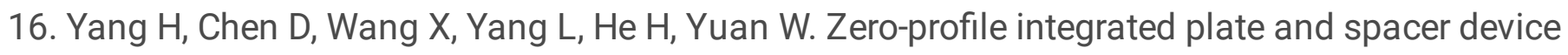
reduces rate of adjacent-level ossification development and dysphagia compared to ACDF with plating and cage system. Arch Orthop Trauma Surg. 2015;135(6):781-7.

17. Vernon H, Mior S. The Neck Disability Index: a study of reliability and validity. J Manipulative Physiol Ther. 1991;14(7):409-15.

18. Scholz M, Reyes PM, Schleicher P, Sawa AG, Baek S, Kandziora F, et al. A new stand-alone cervical anterior interbody fusion device: biomechanical comparison with established anterior cervical fixation devices. Spine. 2009;34(2):156-60.

19. Smith GW, Robinson RA. The treatment of certain cervical-spine disorders by anterior removal of the intervertebral disc and interbody fusion. The Journal of bone and joint surgery American volume. 1958;40-a(3):607 - 24. 
20. Pitzen TR, Chrobok J, Štulik J, Ruffing S, Drumm J, Sova L, et al. Implant complications, fusion, loss of lordosis, and outcome after anterior cervical plating with dynamic or rigid plates: two-year results of a multi-centric, randomized, controlled study. Spine. 2009;34(7):641-6.

21. Song K-J, Lee K-B. A preliminary study of the use of cage and plating for single-segment fusion in degenerative cervical spine disease. Journal of clinical neuroscience. 2006;13(2):181-7.

22. Rhee JM, Yoon T, Riew KD. Cervical radiculopathy. J Am Acad Orthop Surg. 2007;15(8):486-94.

23. Assietti R, Beretta F, Arienta C. Two-level anterior cervical discectomy and cage-assisted fusion without plates. NeuroSurg Focus. 2002;12(1):1-5.

24. Song K-J, Taghavi CE, Lee K-B, Song J-H, Eun J-P. The efficacy of plate construct augmentation versus cage alone in anterior cervical fusion. Spine. 2009;34(26):2886-92.

25. Riley LH III, Skolasky RL, Albert TJ, Vaccaro AR, Heller JG. Dysphagia after anterior cervical decompression and fusion: prevalence and risk factors from a longitudinal cohort study (presented at the 2004 CSRS Meeting). Spine. 2005;30(22):2564-9.

26. Wang Z, Jiang W, Li X, Wang H, Shi J, Chen J, et al. The application of zero-profile anchored spacer in anterior cervical discectomy and fusion. Eur Spine J. 2015;24(1):148-54.

27. Chen $Y$, Chen H, Wu X, Wang X, Lin W, Yuan W. Comparative analysis of clinical outcomes between zero-profile implant and cages with plate fixation in treating multilevel cervical spondilotic myelopathy: a three-year follow-up. Clinical neurology neurosurgery. 2016;144:72-6.

28. Cheung ZB, Gidumal S, White S, Shin J, Phan K, Osman N, et al. Comparison of Anterior Cervical Discectomy and Fusion With a Stand-Alone Interbody Cage Versus a Conventional Cage-Plate Technique: A Systematic Review and Meta-Analysis. Global spine journal. 2019;9(4):446-55.

29. Vaccaro AR, Balderston RA. Anterior plate instrumentation for disorders of the subaxial cervical spine. Clinical orthopaedics and related research. 1997(335):112-21.

30. Sun Z, Liu Z, Hu W, Yang Y, Xiao X, Wang X. Zero-Profile Versus Cage and Plate in Anterior Cervical Discectomy and Fusion with a Minimum 2 Years of Follow-Up: A Meta-Analysis. World neurosurgery. 2018;120:e551-e61.

\section{Figures}




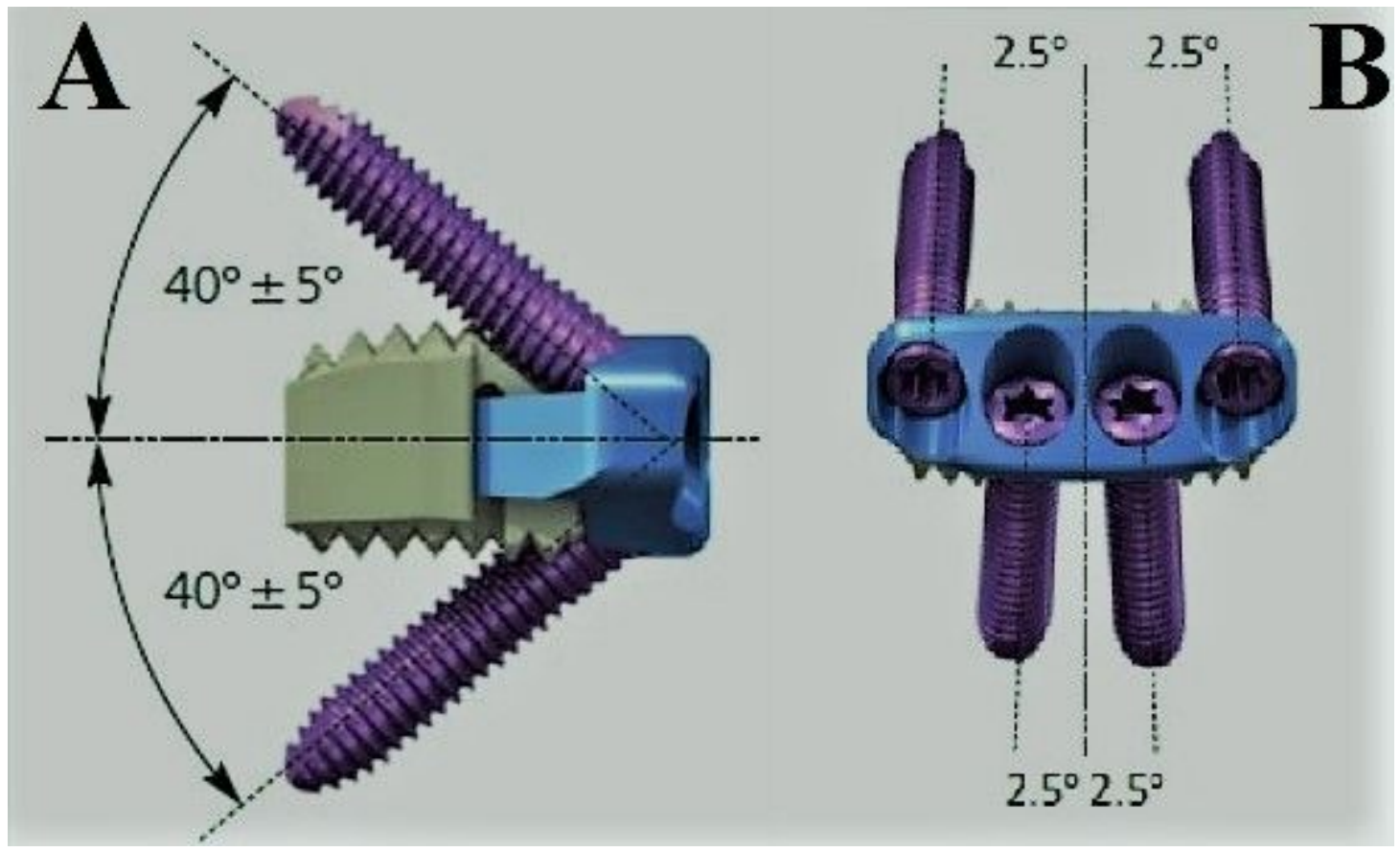

Figure 1

Description of the implant used in the current series [Zero profile cage plate (zero-p; Synthes GmbH, Oberdorf, Switzerland)] A, view from the side, B, view from the front surface. 

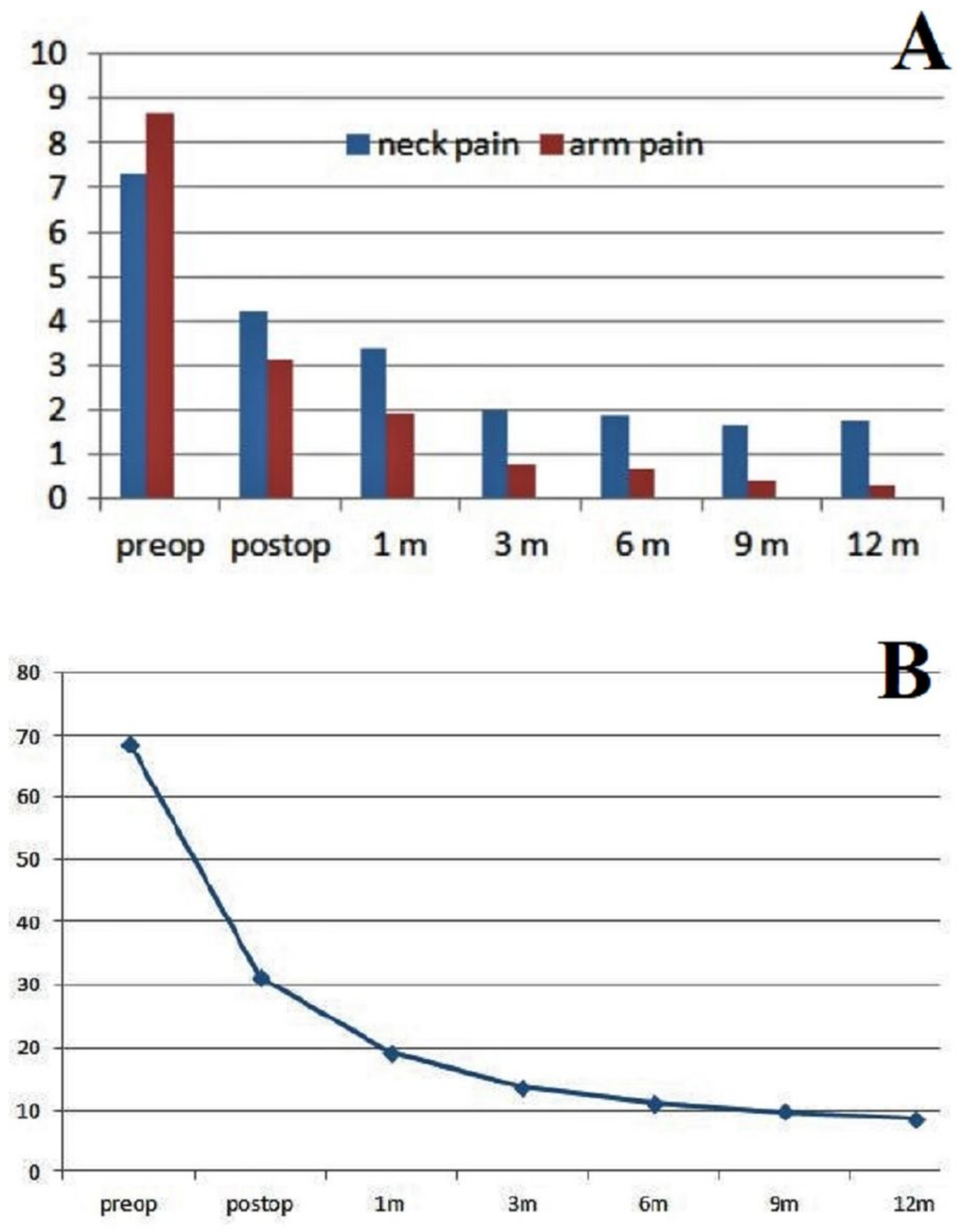

Figure 2

Functional outcomes measured according to VAS and NDI. A, Bar graph representing pre and postoperative arm and neck pain as 0 to 10 VAS over the follow-ups, B, Graphic representing the pre and postoperative changes in NDI scores. (VAS: visual analog scale, NDI: neck disability index). 

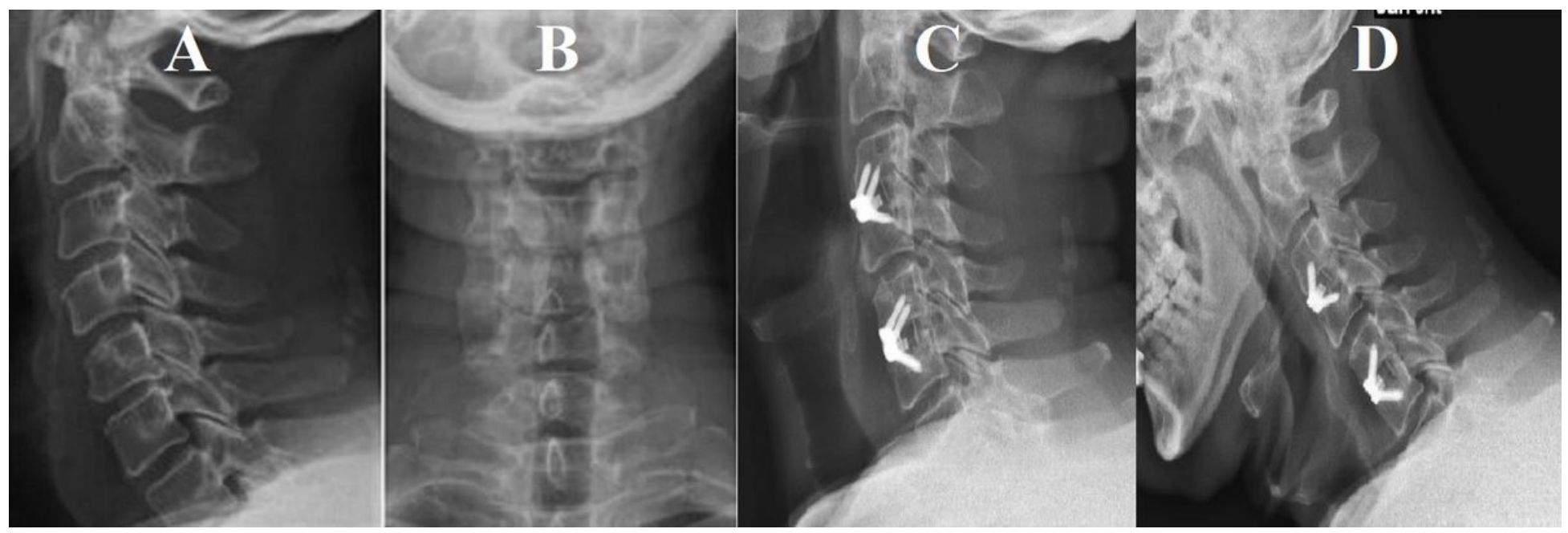

\section{Figure 3}

Postoperative radiographs showing evidence of bony fusion and implant-related complications at 12 months follow-up. (Preoperative radiographs, A: lateral, B: anteroposterior - Postoperative radiographs, C: lateral, D: lateral inflection).
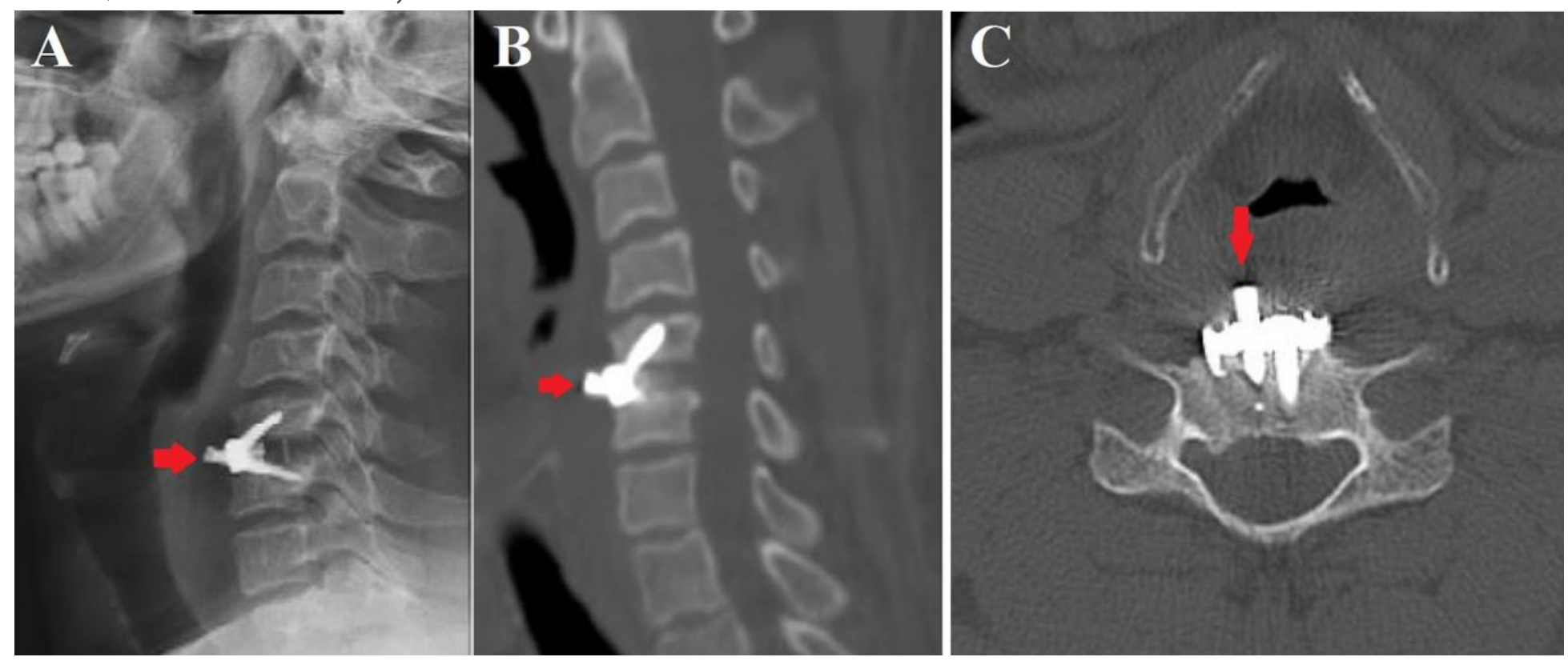

\section{Figure 4}

Screw backing out (red arrowhead) in one patient at three months follow up causing an insidious onset of dysphagia after a free postoperative interval, A: plain radiograph lateral view. Cervical spine CT scan, B: sagittal, C: axial views. 